\title{
A model for the observed periodicity in the Flaring Rate on YY Gem
}

\author{
$\begin{array}{llll}\text { J.G. Doyle } & \text { G.H.J. van den Oord } & \text { C.J. Butler } & \\ & & \text { T. Kiang }\end{array}$
}

\author{
${ }^{1}$ Armagh Observatory, Armagh, N. Ireland \\ ${ }^{2}$ Observatoire de Paris, Meudon, France \\ ${ }^{3}$ Dunsink Observatory, Dublin 15, Ireland
}

\begin{abstract}
Summary: Four flares were observed on the late-type binary YY Gem in March 1988 during a total monitoring time of $408 \mathrm{~min}$. The flares were unusual in that there is a periodicity in their occurrence, being separated by $48 \pm 3 \mathrm{~min}$. Considering the flares to be formed as a stochastic process, we find that the probability of these events occurring by chance is $0.5 \%$. Modelling indicates that for quite reasonable input parameters (e.g. a spot field strength of $1000 \mathrm{G}$ and a filament with mass per unit length of $10^{6} \mathrm{~g} \mathrm{~cm}^{-1}$ ), the flare periodicity can be explained in terms of filament oscillations. The only requirement is that there should be a filament at these heights where the magnetic field drops inversely proportional to the height.
\end{abstract}

\section{Introduction}

Here, we report on observations of flares on the eclipsing binary YY Gem (Castor C = G1 278C), which has a photometric period of 0.8142822 days (van Gent 1931). Both components are classified as dMe1 (Joy and Abt 1974), where the 'e' refers to the Balmer lines being in emission in the 'quiescent' state. Flare activity on this star was first reported by Moffett and Bopp (1971). The flares reported here are unusual in that there seems to be a periodicity in their occurrence. Below we discuss an interpretation of the flare data in terms of filament oscillations.

\section{Observations and data reduction}

The data were taken with a photometer on the University of Hawaii $60 \mathrm{~cm}$ telescope on Mauna Kea from 2 to 7 March 1988. The integration time in the Johnson U-band was 5 secs. The figure below shows a section of U-band data (sky subtracted) in cts/s versus time in U.T. obtained on 6 March 1988. The first event at 7:30 UT is just detectable above the noise, with the last event at 9:59 UT being the largest. The $10-15 \mathrm{~min}$. gaps in the data were times where we were either taking measurements of the sky or a comparison star. 


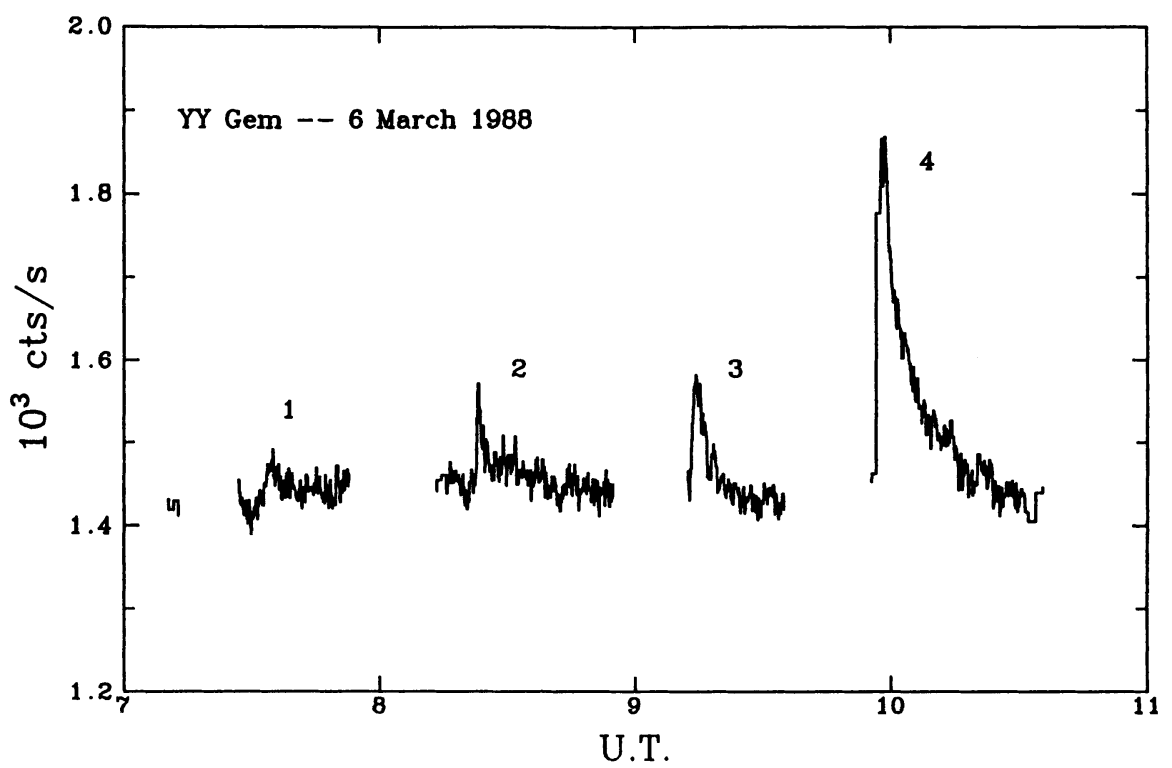

\section{$3 \quad$ Flare statistics}

The most interesting aspect of the flare data is the time-spacing between the peaks of the four events on 6 March 1988, i.e. $48 \pm 3$ mins. Previous authors have looked for such a periodicity in flare occurrence, e.g. Lukatskaya (1976) analysed 782 U-band flares from UV Ceti but found no evidence for a periodicity. Similar, Pettersen (1983) also attempted a correlation between the time-spacing of flares on V780 Tau and their energies, but also obtained a negative result. The reason for such interest is the idea of a "flare reservoir", i.e. an active region has a certain amount of stored energy which is then released either as a single flare or as a multiple of smaller events. Pazzani and Rodono (1981) in a statistical analysis of flare data on three stars, UV Ceti, EQ Peg and YZ Cmi showed that there was a significant departure from Poissonian behaviour in the distribution of time interval between successive flares and the number of events observed within a fixed time interval (these events were described as precursors). The four flares of 6 March 1987 on YY Gem occurred at intervals of 49,51 and $44 \mathrm{~min}$. The question one has to ask, is there a significant periodicity?, or rather assuming flaring to be a Poisson process, what is the probability of four flares occurring with more regular spacings than the above? Applying a detailed statistical analysis we find that the probability of the four flares arising out of a chance process is rather small $(\leq 0.5 \%)$, and so we conclude that there is a significant periodicity in their occurrence (a complete description of the statistical analysis is given in a paper to be published in Astron. Astrophys.) 


\section{A model}

The observed periodicity of 48 minutes is long compared to MHD time-scales, e.g. for a loop of length $10^{10} \mathrm{~cm}$ and Alfven speed of $300 \mathrm{~km} \mathrm{~s}^{-1}$, the Alfven travel time is only 6 minutes. It is therefore unlikely that the flares are triggered by an MHD process inside a coronal loop. We therefore consider the trigger mechanism to be related to filament oscillations.

The net force $\mathrm{F}$ on a filament in vertical direction and per unit length of the filament is given by

$$
\lambda \frac{d^{2} h}{d t^{2}}=F(h)
$$

where $\mathrm{h}$ is the height of the filament above the photosphere and $\lambda$ the mass of the filament per unit length. If the filament is in equilibrium at position $h_{o}$, we have $F\left(h_{o}\right)=0$. Suppose that the filament is displaced over a small distance $\epsilon$, e.g. by a passing shock wave, then we can write $h=h_{o}+\epsilon$. Substituting this in equ. (1) and making a Taylor expansion for $\epsilon$ gives

This can be rewritten as

$$
\lambda \frac{d^{2} \epsilon}{d t^{2}}=\left.\epsilon \frac{d F}{d h}\right|_{h_{o}}
$$

$$
\frac{d^{2} \epsilon}{d t^{2}}+\omega^{2} \epsilon=0
$$

with

$$
\omega^{2}=-\left.\frac{1}{\lambda} \frac{d F}{d h}\right|_{h_{\circ}}
$$

Under the condition $(d F / d h)_{h_{o}}<0$, we see that the filament behaves as a harmonic oscillator with period $P=2 \pi / \omega$. The net force $F$ on a filament is given by van Tend and Kuperus (1978) as

$$
F(h)=\left(\frac{l}{c}\right)^{2} \frac{1}{h}-\left(\frac{l}{c}\right) B(h)-\lambda g
$$

with $l$ the current in the filament (in statamps), $B(h)$ the active region potential field strength (in Gauss) as a function of height and $g$ the gravitational acceleration. This expression is valid for heights $h$ smaller than a stellar radius. The first term on the righthand side accounts for the Lorentz force between the filament current and the currents induced in the photosphere by the magnetic field of the filament (see van Tend and Kuperus 1978 for further details). The terms are the Lorentz force on the filament current by the active region magnetic field and gravitational force on the filament.

In order to calculate the oscillation period of the filament at a specific height $h$ in the corona we need to evaluate $d F / d h$ for $F(h)=0$. From equ. (5) we conclude that the current $l$ required at height $h$ for the filament to be in equilibrium (i.e. $F(h)=0$ ) is

$$
\left(\frac{l}{c}\right)=\frac{1}{2} h B(h)+\left[\left(\frac{1}{2} h B(h)\right)^{2}+\lambda g h\right]^{1 / 2}
$$

The gradient of the force is then given by

$$
\frac{d F}{d h}=-\left(\frac{l}{c}\right)^{2} \frac{1}{h^{2}}-\left(\frac{l}{c}\right) \frac{d B}{d h}
$$


This then allows us to determine the oscillation period of the system as a function of the equilibrium position. We assume that the magnetic field strength in the stellar spots is $B_{o} \approx 1000$ Gauss and the mass per unit length of the filament $\lambda=10^{6} \mathrm{gram} \mathrm{cm}^{-1}$ (see van Tend and Kuperus). Taking the masses of the components to be $0.6 M_{\odot}$ and the radii $0.6 R_{\odot}$, this results in a gravitational acceleration of $g_{\odot} / 0.6$. This gives for $D / 2<h<D$ (where $D$ is the active region size)

$$
\omega^{2}=\frac{g}{h}=\frac{2 g}{D}\left(\frac{D / 2}{h}\right)
$$

For heights corresponding to $D / 2<h<D$, the period of the oscillations is given by

$$
P=\frac{2 \pi}{\omega}=2 \pi\left(\frac{h}{g}\right)^{1 / 2}=70.8\left(\frac{D}{R_{*}}\right)^{1 / 2}\left(\frac{2 h}{D}\right)^{1 / 2} \text { minutes }
$$

This time scale is indeed comparable to the observed period. Taking $P=48$ minutes, we find for the ratio of the size of the active region to stellar radius

$$
\left(\frac{D}{R_{*}}\right)=0.46\left(\frac{D}{2 h}\right)
$$

For $D / 2<h<D$ we find $0.23<\left(D / R_{*}\right)<0.46$ or $9.610^{9} \mathrm{~cm}<D<19.210^{9} \mathrm{~cm}$.

For $h<D / 2$, the oscillation frequency is $\omega \approx 1 s^{-1}$. The reason for this extremely short frequency is that close to spots, gravity in unimportant and stability is maintained by the Lorentz force of the photospheric surface currents and the Lorentz force of the active region.

These results indicate that for quite reasonable input parameters $\left(B_{o} \approx 1 k G, \lambda=\right.$ $10^{6} \mathrm{~g} \mathrm{~cm}^{-1}$ ), filament oscillations can be expected. The only requirement being that there should be a filament at these heights where the active region varies like $1 / h$.

\section{References}

Joy, A.H., Abt, H.A.: 1974, Astrophys. J. Supp. 29,1

Lukatskaya, F.L.: 1976, Soviet Astron. Lett. 2,61

Moffett, T.J., Bopp, B.W.: 1971, Astrophys. J. Lett. 168,L117

Pazzani, V., Rodono, M.: 1981, Astrophys. \& Space Sci. 77,347

Pettersen, B.R.: 1983, Astron. Astrophys. 120,192

van Gent, H.: 1931, B.A.N. 6,99

van Tend, W., Kuperus, M.: 1978, Solar Phys. 59,115 